\section{Design animal studies better}

Steve Perrin argues that animal models of human disease should be better characterized to avoid false-positive results (Nature 507, 423-425; 2014). Poor design, execution and reporting of studies are pervasive contributors as well.

For example, randomization and blinding in animal studies is pitifully infrequent (see, for instance, J. P. Ioannidis et al. Lancet 383, 166-175; 2014). Randomly allocating animals to experimental and control groups makes the experimental groups as similar as possible in other respects. Blinding promotes comparable handling and measurement by experimenters, and publicly preregistering animal-study protocols and the outcomes to be measured would identify and reduce bias in reporting results.

Creating largely homogeneous experiments aids reproducibility and boosts statistical power, but has a cost of generalizability: the few drugs that have translated successfully from animals are effective across a broad range of circumstances (see, for example, E. S. Sena et al. J. Cereb. Blood Flow Metab. 30, 1905-1913; 2010).

Funders and ethics committees need to ensure that study designs include these features, and journals should make them criteria for publication if they have not done so already. Malcolm R. Macleod ${ }^{\star}$ University of Edinburgh, UK. malcolm.macleod@ed.ac.uk ${ }^{\star}$ On behalf of 6 co-authors (see go.nature.com/js4uzj for full list).

\section{Investment suggests RNAi is on the up}

If pharmaceutical investment is a measure of the prospects for therapeutics that use RNAinterference (RNAi) technology for gene silencing (Nature 508, $443 ; 2014)$, on balance, things are looking up.
Yes, Novartis shuttered its RNAi effort and Merck sold its RNAi programme to Alnylam Pharmaceuticals. These were the right decisions: Novartis and Merck are both accomplished companies, but their decade-long efforts in RNAi failed to yield any clinical programmes.

What you did not mention was Genzyme's investment in Alnylam in early 2014 - one of the largest and most significant strategic transactions in recent biotech history. Genzyme, a Sanofi company, purchased a $12 \%$ stake in our company in return for broad product rights in the field of rare diseases in regions outside North America and western Europe.

Companies such as Genzyme do well to partner with technology innovators such as Alnylam for access to their products, not to the technology platform. This is how drug companies ultimately had success with recombinant DNA and monoclonal antibodies. The same story could be emerging with RNAi.

John Maraganore Alnylam Pharmaceuticals, Cambridge, Massachusetts, USA. jmaraganore@alnylam.com

\section{Mine centuries-old citizen science}

Historical data on biodiversity would be valuable for investigating the long-term impact of human activities. Contrary to popular belief, such data have been widely collected for several hundred years through initiatives that would today be described as 'citizen science'.

For example, in the late sixteenth century, the Spanish government distributed questionnaires known as relaciones topográficas to each village, with local inhabitants providing a compilation of natural-history knowledge. The 637 questionnaires that survive include information on some 190 species of wild animals and plants, gleaned from more than 4,300 individual records.

Also, the 16-volume geographical dictionary edited by the statistician and politician Pascual Madoz in the midnineteenth century contains information on most Spanish population centres, rivers and geographical landmarks. Madoz's enterprise involved the collaboration of more than 1,000 citizens and provided several thousand records of wild plants and animals, as yet unused.

Similar historical data sets also exist from China, most European countries and their former colonies. These can be biased towards socio-economically important species, but this bias can help to minimize misidentifications, for instance.

We call for an international, multidisciplinary effort (including historians, linguistic experts, geographers and biologists) to locate, compile and contextualize these invaluable historical data for incorporation into global biodiversity databases.

Miguel Clavero, Eloy Revilla Doñana Biological Station CSIC, Seville, Spain. miguelclavero@ebd.csic.es

\section{Moon mapped by an artist's impression}

Thomas Harriot mapped the Moon before Galileo (J. Rampling Nature 508, 39-40; 2014). Unlike Galileo, he did not interpret what he saw as craters, mountains and valleys (see S. Pumfrey Notes Rec. R. Soc. 63, 163-168; 2009).

Galileo's experience in the visual arts gave him a theoretical background in mathematical perspective (see, for example, S. Y. Edgerton The Heritage of Giotto's Geometry 233-239, Cornell Univ. Press, 1991; and S. E. Booth and A. Van Helden Sci. Context 13, 463-486; 2000).
This included using shadows to determine the volume and location of an object. Observing the Moon's surface with his perspicillum, later named telescope, Galileo's training as a draftsman led him to interpret variations in light on the surface as shadows produced by the Sun on a complex topography.

Harriot did not grasp the significance of these dark areas. He interpreted them simply as surface markings that he used to 'map' the Moon. Art and science have sometimes benefited each other in ways that we fail to appreciate.

José Rafael Martínez Enríquez National Autonomous University of Mexico, Mexico City, Mexico. enriquez@unam.mx

\section{Experimentation needs theory, too}

John Skoyles emphasizes the importance of practical experimental work for the developing scientist (Nature $508,319 ; 2014)$. But theory is crucial too - for interpreting the results and for advancing research.

In 1928, British physicist Paul Dirac came up with his equation, which predicted the existence of the positron. The particle was discovered independently four years later by US physicist Carl Anderson at the California Institute of Technology in Pasadena. This is a good example of "theory guides; experiment decides" (the motto of analytical chemist Izaak Maurits Kolthoff).

Or as the astrophysicist Arthur Stanley Eddington declared: "It is also a good rule not to put overmuch confidence in the observational results ... until they are confirmed by theory."

Perhaps the last word should come from Soviet physicist Peter Leonidovich Kapitza. He said: "Theory is a good thing, but a good experiment is forever." Min-Liang Wong National Chung-Hsing University, Taiwan. mlwong@dragon.nchu.edu.tw 